\title{
Efficiency, capacity, compensation, maintenance, plasticity: emerging concepts in cognitive reserve
}

\author{
Daniel Barulli and Yaakov Stern
}

Cognitive Neuroscience Division, Department of Neurology, Columbia University College of Physicians and Surgeons, New York, USA

\begin{abstract}
Cognitive reserve (CR) is a concept meant to account for the frequent discrepancy between an individual's measured level of brain pathology and her expected cognitive performance. It is particularly important within the context of aging and dementia, but has wider applicability to all forms of brain damage. As such, it has intimate links to related compensatory and neuroprotective concepts, as well as to the related notion of brain reserve. In this article, we introduce the concept of cognitive reserve and explicate its potential cognitive and neural implementation. We conclude that cognitive reserve is compatible and complementary with many related concepts, but that each much draw sharper conceptual boundaries in order to truly explain preserved cognitive function in the face of aging or brain damage.
\end{abstract}

\section{The reserve concept}

CR (see Glossary) has been proposed to account for the frequent discrepancy between a person's underlying level of brain pathology (or age-related changes) and the observed functional and/or cognitive deficits that are expected to result from that pathology [1,2]. There is extensive epidemiological and experimental evidence for the existence of such reserve: life exposures, such as educational and occupational attainment, and engagement in leisure and social activities have each been associated with decreased risk of developing dementia [3-6], more successful aging [7], and reduced clinical changes in several other conditions, including traumatic brain injuries [8], Parkinson's disease (PD) [9], multiple sclerosis (MS) [10], and HIV-related dementia [11] (Figure 1).

The status of CR as a concept has been debated vis-à-vis other related concepts, such as brain reserve (BR) [12] and more recently brain maintenance (BM) [13]. Several other related concepts have also been proposed [14]. In this review, we discuss recent work on these and related con-

Corresponding author: Stern, Y. (ys11@columbia.edu).

Keywords: cognitive reserve; aging; Alzheimer's disease; imaging; epidemiology.

1364-6613/\$ - see front matter

(C) 2013 Elsevier Ltd. All rights reserved. http://dx.doi.org/10.1016/j.tics.2013.08.012 cepts, and attempt to delineate the subtle distinctions between them. We argue that, although the concepts differ in important respects, they are complementary as opposed to competing.

\section{Models of reserve}

Below we outline some of the dominant theories of preserved cognitive function in the face of advanced age, dementia, and/or brain damage. These theories focus either on compensatory mechanisms (emphasizing adaptations to diminished function or impaired brain structure), neuroprotective mechanisms (emphasizing factors which prevent diminished function and impaired structure), or some combination of both. The main models we discuss are $\mathrm{BR}, \mathrm{CR}, \mathrm{BM}$, and neurocognitive scaffolding.

\section{Glossary}

Brain reserve (BR): differences in brain size and other quantitative aspects of the brain that explain differential susceptibility to functional impairment in the presence of pathology or other neurological insult.

Cognitive reserve (CR): differences in cognitive processes as a function of lifetime intellectual activities and other environmental factors that explain differential susceptibility to functional impairment in the presence of pathology or other neurological insult.

Neural reserve: one proposed neural basis of cognitive reserve that involves cognitive networks used by unimpaired individuals. Individual differences in network efficiency/capacity or the use of alternative strategies may provide reserve against the impact of brain changes.

Efficiency: the degree to which a task-related brain network must become activated in order to accomplish a given task.

Capacity: the degree to which a task-related brain network can be activated maximally to keep performing a task even in the face of increasing demands. Neural compensation: one proposed neural basis cognitive reserve involving the utilization of alternative networks not typically used by healthy individuals in order to maintain or improve cognitive performance.

Brain maintenance (BM): individual differences in susceptibility to pathology, particularly in the context of aging; whereas reserve theories emphasize compensatory mechanisms, maintenance theories emphasize neuroprotective mechanisms.

Scaffolding theory of aging and cognition (STAC): scaffolding is the recruitment of additional neural circuits or networks when the primary networks have become inefficient or damaged due to age, pathology, or even some normal task-related challenge. This process is in theory a general and life-long property of the brain.

Compensation-related utilization of neural circuits hypothesis (CRUNCH): the theory that, as a task becomes more difficult, a network will be recruited to an increasing degree. At some point, increased difficulty overwhelms the network, which ceases to function effectively.

Cognitive flexibility: the capacity to achieve best performance on a particular task, given the range of ability currently supported by the brain's underlying neuroanatomical structure; transient fluctuations in function that do not result in long-term structural or anatomical changes fall under this umbrella. 


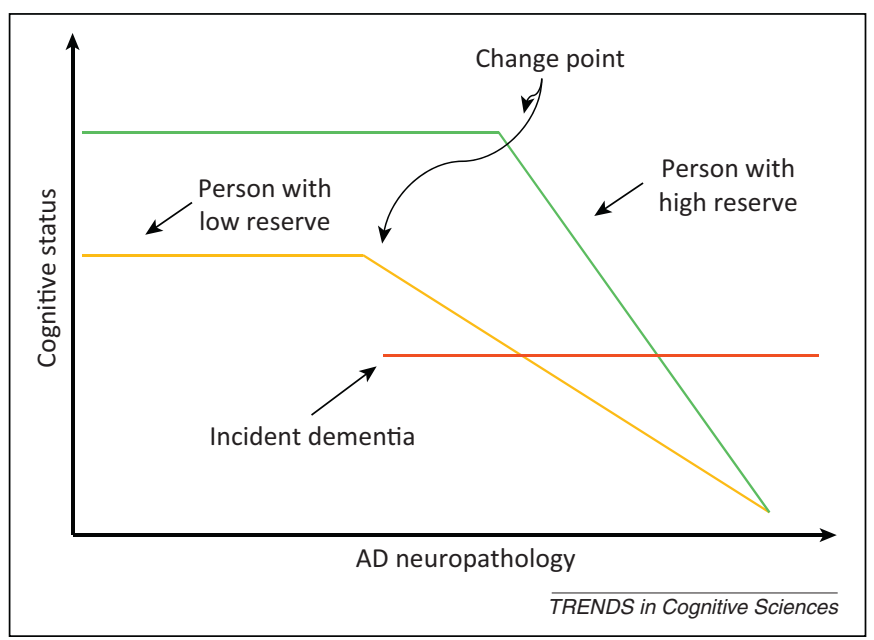

Figure 1. Representation of how CR may mediate between AD pathology and its clinical expression based on epidemiological and imaging studies. The $x$-axis represents $A D$ pathology, slowly increasing over time. The $y$-axis represents cognitive function. We assume that $A D$ pathology increases over time at the same rate in two individuals with high and low reserve. The amount of pathology needed before cognitive function is affected is greater with higher CR, leading to a later change point $[60,61]$. It follows that more pathology will be needed for the person with higher $C R$ to meet clinical diagnostic criteria for $A D$, thus delaying the onset of the disease. Also, at any level of cognitive performance, AD pathology will be more severe in the individual with higher CR $[27,62]$. Once cognitive decline begins, it is more rapid in the person with higher CR $[61,63]$.

\section{Brain reserve}

The notion of BR posits that differential susceptibility to brain damage or pathology is a function of $i$ ) the extent of the brain damage and ii) a purely quantitative measure of brain reserve capacity (BRC) (such as the overall size of the brain, the number of neurons, the number of synapses, etc.) [12]. When pathology reduces BRC beyond a certain threshold, functional decline occurs. This can explain how pathology that is relatively equal between two people can yield differing functional manifestations (e.g., two people with equal levels of $\mathrm{AD}$ pathology, such as amyloid or tau deposition, can perform vastly differently on cognitive tests).

This conceptualization of BR can be considered a passive, threshold model, because once a certain ratio of pathological quantity to brain quantity is reached, functional impairment is inevitable. The general threshold model can be applied to virtually any pathology the brain may face. To take one example, a higher number of large pyramidal neurons in the cortex has been suggested as one potential quantitative measure that could predict differences in the rate of functional impairment given the same amount of $\mathrm{AD}$ pathology [15]. Most other related studies of BR typically use more generalized measures of BRC, such as total intracranial volume. However, although associations between head size and resistance to dementia have been found [16], often more sophisticated analyses that control for genetic factors, such as the apolipoprotein $E$ (Apo-E) gene, have dampened this association [17]. Moreover, the link between head circumference and dementia is often significant only towards the extremely low ranges of the former [18].

More nuanced potential measures of BR are more feasible. For instance, imaging measures, such as diffusion tensor imaging (DTI), provide a new quantitative measure that can be used in the traditional threshold model (see, e.g., [19,20]). Eventually, microstructural anatomical differences in measures such as dendritic spine length, dendritic density, or synaptic proteins may be employed $[21,22]$. Although in practice these measures are difficult to obtain in humans, except in autopsy studies, their homologues in animals can be examined using histology and their effects on animal behavior can be observed [23].

\section{Cognitive reserve}

In contrast to BR, CR can be considered to be an 'active' model, in that the threshold for functional decline is not fixed by quantitative brain measures, but can be altered based on experience. Thus, individuals with the same amount of BRC can have different levels of CR [1]. The CR model posits that cognitive processes are crucial for explaining the differences between someone who is functionally impaired and someone who is not, despite equal brain changes or pathology. These cognitive processes consist of differences in cognitive efficiency, capacity, or flexibility that are shaped by life experiences. Thus CR is 'active' in two senses: i) it relies on current neural activity to explain functional differences much more heavily than $\mathrm{BR}$ and ii) it suggests that current neural activity is shaped by disparate cognitive exposures/activities throughout the lifespan.

$\mathrm{CR}$ is often estimated using proxy variables for lifetime exposures and cognitive activity: years of education, measures of crystallized intelligence, such as vocabulary or knowledge, literacy level, number of intellectually stimulating leisure activities, degree of occupational complexity, and socioeconomic status are all commonly used to create an estimate of CR [1]. There are important methodological issues to consider in combining these CR proxies [24]. In addition, an alternative CR measure has been proposed that comprises the variance in cognitive performance not explained by socio-demographic measures and measures of brain pathology [25].

Recent evidence for the role of CR (as inferred via a number of proxies) in modulating the cognitive effects of pathology and normal aging is vast. Here, we provide a few representative recent studies as examples. In one study, the relationship between low plasma beta-amyloid (A $\beta$ ) and cognitive performance was modulated by CR: elders with higher education showed a weaker association [26]. Similarly, higher education has been associated with diminished fludeoxyglucose positron emission tomography (FDG-PET) activation in subjects positive for cerebrospinal fluid $\mathrm{A} \beta$, suggesting a compensatory role for CR [27]. Beyond $\mathrm{AD}$, higher education has been associated with slower transition from mild cognitive impairment (MCI) to dementia in PD [9]. Similarly, higher CR (as measured using reading score on the WRAT-3) is protective against cognitive decline in women following chemotherapy treatment for breast cancer [28]. In the context of normal aging, higher CR (measured with educational and occupational history, as well as verbal IQ) was associated with lower functional MRI activation in right inferior frontal cortex during a working memory task (consistent with increased network efficiency - see below), as well as with decreased brain volumes in older adults with MCI and AD (consistent 
with more preservation of function in the presence of brain pathology) [29]. In the same vein, the strength of the blood oxygen level-dependent (BOLD) signal in task-related areas as-yet unaffected by $\mathrm{AD}$ pathology or gray matter atrophy was found to be positively correlated with $\mathrm{CR}$ proxies (again using occupational-educational history and verbal IQ, plus a measure of leisure activities) in $\mathrm{MCI}$ and $\mathrm{AD}$ subjects, but negatively in healthy subjects [30]. These findings suggest that CR operates in a compensatory manner even before pathology begins to diminish function in a particular area [30].

Because CR is a cognitive concept, it is important to identify its neural implementation. Stern [31] posited two neural mechanisms: neural reserve and neural compensation (Figure 2). Neural reserve addresses the cognitive networks that have developed over the lifespan as a function of innate capacity and lifetime exposures. Someone with higher neural reserve, may therefore have more efficient cognitive networks (i.e., networks that need to activate to a smaller degree than a less efficient network in order to perform the same task at a comparable level of performance), higher capacity networks (i.e., ones that can activate to a greater degree given increasing task difficulty), or greater flexibility in network selection. Thus, neural reserve encapsulates most of the differences observed between healthy individuals on cognitive tasks and posits that these differences may account for differential susceptibility to brain changes or pathology. Interestingly, recent theoretical models also equate higher intelligence to higher neural efficiency [32]. The neural reserve concept is compatible with Fabiani's view of normal healthy aging as the continuation of processes that are already present earlier in life and which continually sculpt and transform the brain [33].
Neural compensation refers to situations where pathology (or age-related changes) afflicts primary task-related networks and necessitates the use of additional, compensatory networks to accomplish the same task. Individuals with higher levels of CR may be more capable of drawing on alternative neural networks in the face of brain changes in order to maintain function.

Although BR and CR began as very distinct concepts, much recent work that demonstrates the plasticity of the brain itself in response to experience suggests that the boundaries between the two models should be softened. In animal studies, voluntary aerobic exercise has been associated with dramatic structural changes, including increased neurogenesis in the dentate gyrus of the hippocampus [34] and upregulation of brain-derived neurotrophic factor (BDNF) [35]. Similarly, cognitive experiences have been associated with similar brain changes $[22,36]$. Furthermore, 'cognitive' training can result in focal volume changes in areas relevant to task demand, which suggests that both long-term intellectual stimulation, as well as focused cognitive interventions can produce structural alterations [23,37] (Box 1).

These structural changes are not confined to animal models. Many cross-sectional studies have also suggested that volumetric changes occur in humans following years of intellectual stimulation associated with higher education [38], specialized occupation [39], literacy [40], and so on. Caution is warranted against uncritically accepting this conclusion, given the vague causal directionality of the association between enriched experience and more intact brain structure [22]. Nonetheless, prospective studies support this idea. Increased hippocampal size in humans following a 6-month aerobic exercise intervention has been reported [41]. Moreover, three months of training on

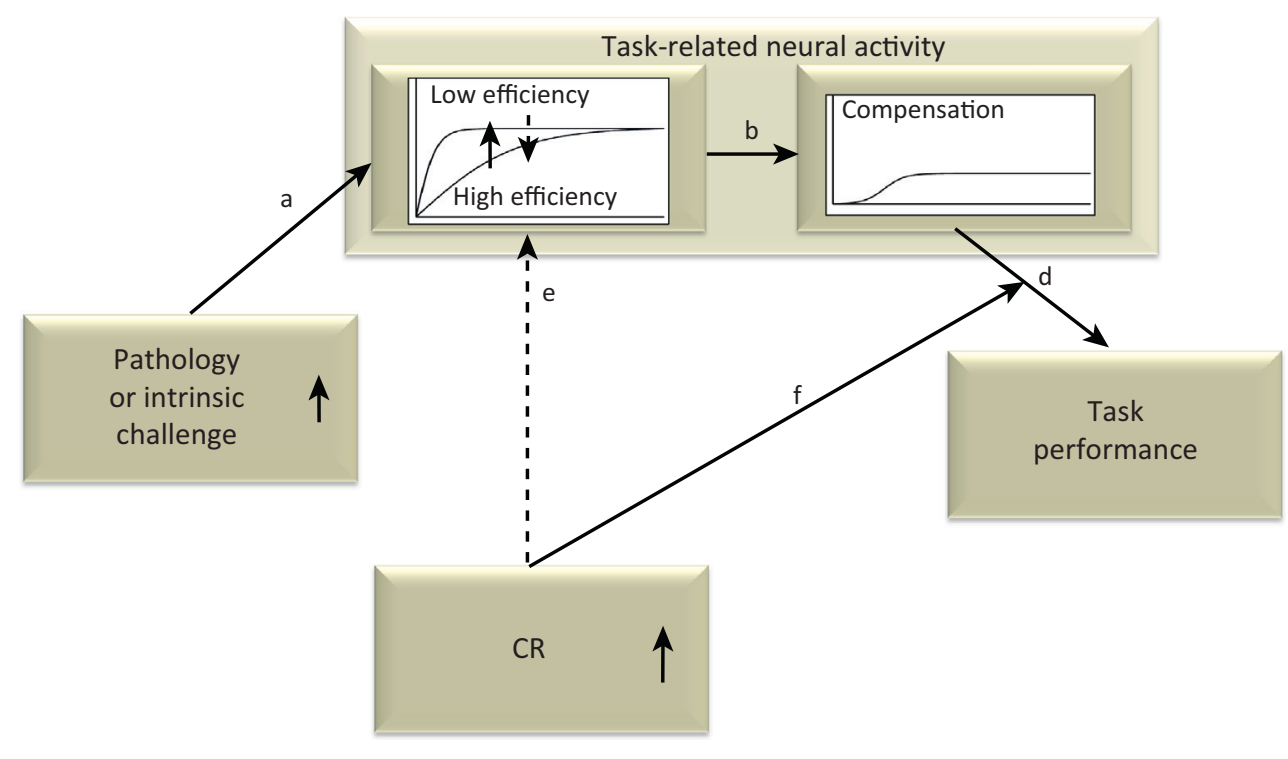

TRENDS in Cognitive Sciences

Figure 2. Possible instantiation of CR via neural reserve and neural compensation. One study [64] using this model in the context of a working memory task observed decreasing network efficiency with volume loss within the network (path a). However, this decreasing efficiency was mitigated by higher measured CR (path e), consistent with the concept of neural reserve. Neural compensation was also observed via a second network expressed by older but not younger adults when efficiency was sufficiently impaired in the first network (path b). More activation in the compensatory network was associated with diminished task performance (path d), which demonstrates that compensation need not always improve performance but may only maintain it, albeit at a lower level. Higher measured CR moderated the detrimental effect of the compensatory network on performance (path f), which suggests that individuals with higher CR may recruit additional networks not directly captured by taskrelated activation. 


\section{Box 1. Implications for intervention}

Epidemiological data indicate that lifelong exposures are associated with more successful aging $[44,56,65]$. However, the transition from observation to intervention is not straightforward. It is unknown whether short-term engagement in any set of activities is sufficient to impart reserve; it is possible that reserve accrues only over long periods of time. Also, although intervention strategies can be guided by epidemiology, their optimal instantiation is not straightforward.

Aerobic exercise has well-documented benefits and has resulted in improved performance on cognitive tests, especially in executive function [66]. A recent meta-analysis of 29 studies concluded that aerobic exercise is associated with improvements in various cognitive domains, such as processing speed, executive function, memory, and attention [67].

Cognitive or behavioral interventions have yielded more mixed results. A large-scale study found some improvements on trained domains, but no transfer of training across domains or improvement in activities of daily living. Studies that have immersed subjects in complex gameplay are also promising. Playing a complex roleplaying game was associated with improved performance on a wide range of cognitive tasks [68] and playing the Space Fortress task with emphasis-change training was associated with improved working memory [69]. Although evidence of genuine transfer-of-training effects remains slim and improvements in day-to-day cognitive functions are elusive [70], one study found that training on a working

juggling skills was associated with increases in left intraparietal sulcus and mid-temporal cortical grey matter [42]. Similarly, learning the layout of London was associated with gray matter increases in trainee taxi drivers, specifically in the hippocampus [43]. There are even suggestions that higher CR may be associated with reduced pathologies, such as reduced rate of hippocampal reduction in aging [44] and lower $A \beta$ deposition [45]. The latter observations suggest strongly that such exposures associated with CR may not only help the brain adapt to structural changes, but it may also help prevent those changes to begin with.

\section{Brain maintenance}

Similar to BR models is the notion of BM [13]. Rather than addressing differential susceptibility to impairment given equal levels of pathology, maintenance models instead ask which factors may protect against pathology or age-related changes, and posit that there are certain genetic factors and favorable life experiences that imbue people with a capacity to resist undergoing these changes. It is an open question whether BM models could be extended to predict that certain individuals are better than others at resisting not only the advent of pathological processes, but also resisting the harmful biological effects of the normal aging process itself. Disentangling the effects of CR and BM on levels of functional impairment is difficult due to practical challenges in identifying and measuring brain changes associated with aging or disease processes. In addition, this approach does not explain variability of functioning in the face of identified brain pathologies such as stroke, $A D$, MS or white matter hyperintensities, nor does it account for recent empirical observations that much age-related cognitive decline is unaccounted for by common neuropathologies [46]. Moreover, there is direct evidence that CR proxies can operate in a compensatory way and not in a neuroprotective way as BM predicts. For instance, Brayne et al. [47] found that years of education were not associated memory task generalized to improvements in measures of fluid intelligence [71]. Moreover, speed of processing training has been shown to lead to increased driving mobility for elder subjects [72].

Cognitive training has also been shown to induce structural changes. Training on several cognitive domains improved white matter (WM) microstructure in both younger and older healthy subjects [73]. Intensive memory training resulted in changes to gray matter volume and WM integrity in elders, and these increases correlated with improved memory performance $[74,75]$. Changes in the structural integrity of WM have been induced with cognitive and visuomotor training [4,76], and video-game playing [77]. Other studies of training on working memory [78], mirror-reading [53], and Morse code [79] have also resulted in structural changes. Such changes may happen very quickly, even after just two hours of category learning [80] or spatial learning [81].

Although epidemiological data suggest that life exposures can enhance reserve, the exact 'recipe' for intervention remains unknown. The most meaningful endpoints for intervention in elders would be slowed rate of age-related cognitive decline or reduced risk of developing $A D$. Controlled studies that use such endpoints will undoubtedly be very expensive and will have to be conducted over long periods of time. Most likely, these studies should use multiple intervention strategies including exercise, cognitive stimulation, and social stimulation.

with neurodegeneration or vascular pathology themselves, but they did moderate the effects of such pathologies on clinical expression. Nonetheless, more direct studies are needed to disentangle the effects of BM from those of reserve theories.

The BM account has intimate links with research into neuroplasticity in animal models, but these two research strands are starkly different in that, whereas one looks explicitly at which factors are associated with the absence of any structural changes, the other looks at which factors facilitate either i) beneficial structural changes (e.g., $[42,48]$ ), or ii) compensatory functional responses, given harmful structural changes (e.g., [49]). The neural reserve and neural compensation implementations of CR are highly related to more recent notions of compensation emerging from the neuroplasticity literature conducted largely with animal studies, but also from observations in human subjects (e.g. [50]; Box 2).

\section{Bridging cognitive reserve and brain reserve}

Another approach taken in a review by Lövdén et al. [51] creates a bridge between $\mathrm{CR}$ and $\mathrm{BR}$. It suggests that people use knowledge-based strategies (which can be considered to be within their current range of cognitive flexibility) to perform tasks, but when faced with a prolonged mismatch between functional supply and challenge (of either the intrinsic or extrinsic nature), the brain itself must exhibit plastic and compensatory alterations that may result in a mechanism akin to neural compensation. When the brain is confronted by a challenge it relies on compensatory network activations to maintain performance; and although these compensatory networks could be less efficient at performing a given task than the primary networks used, without the additional activation performance would be severely impaired. Eventually, these challenges prompt changes in the brain itself, which Lövdén et al. define as true plasticity. When this happens, it seems plausible that more 'process-based' rather than 


\section{Box 2. Using imaging to explore the neural basis of cognitive reserve}

Functional imaging has strengths and weaknesses for evaluating the neural basis of CR. It is not ideal for identifying networks that underlie alternate problem-solution strategies because imaging analyses are more attuned to common rather than different patterns of activation within a group of people. However, functional imaging can be used to probe individual differences in task-related activation as a function of task performance and CR proxies. Consideration of the degree of pathology aids in exploring how some individuals cope with pathology better than others [82].

Neural reserve would predict that individuals with and without a given pathology use the same brain networks, albeit with differing efficiency or capacity. It is important to consider that task-related activation can increase with task difficulty. Because aging or brain changes increase subjective task difficulty and reduce brain-network efficiency, it is common to observe greater task-related activation in more affected than less affected individuals [29]. However, at higher levels of task demand, there can be greater activation in more intact individuals, because of greater network capacity [58]. Controlling for subjective task demand can produce equivalent levels of task-related activation in young and old individuals [83]. The compensationrelated utilization of neural network hypothesis (CRUNCH) [50] encompasses these findings, with the additional observation that activation in more affected individuals might actually decline in

knowledge-based mechanisms (i.e., compensatory mechanisms based on implicit improvements in abilities such as working memory rather than ones based on using explicit strategies such as mnemonics) begin to underlie performance differences. Indeed, Shing et al. [52] demonstrated that some cognitive domains (such as episodic memory) can be broken down into strategic and process-based components (Box 3).

The scaffolding theory of aging and cognition (STAC) Similar to Lövdén et al. [51], Park and Reuter-Lorenz [14] reason that compensatory mechanisms reflect a general feature of the brain that they call scaffolding: the ability to adapt to structural alterations (and task-induced functional limitations) by engaging in functional reorganization that is, developing and/or relying on compensatory networks when the primary networks are no longer efficient at performing some task. This can happen either because more specialized networks are recruited, which accomplish the task better (an instance of scaffolding that happens response to increased task difficulty, perhaps reflecting overwhelming of their networks' capacity. Because young and old use the same networks in this scheme, Stern [2] would not invoke the concept of compensation here.

Neural compensation would be invoked when the affected individuals engage networks not typically used by unaffected individuals. Generic models for compensation in aging include PASA [84] and HAROLD [85]. These models assume that compensatory activation is associated with better performance, with some support [86]. The actual implementation of compensation will depend closely on the networks that underlie the functions in question, and possible supporting networks. Studies have also demonstrated compensatory activation that is not associated with better performance $[54,64]$. In this case, the use of an alternate network may maintain as opposed to improve performance. There is also the possibility of a 'generic' CR network that supports multiple functions [87].

These observations of functional activation patterns consistent with neural reserve and neural compensation can be tied to the CR model when it can be shown that in the face of a comparable amount of brain pathology individuals expected to have higher CR can maintain greater network efficiency or capacity, can compensate in an advantageous way, or can avoid resorting to a less advantageous alternate networks.

during learning) or because the primary networks are being subjected to damage of any kind that renders them suboptimal (an instance of scaffolding that happens with age-related deterioration). Thus, age-related brain changes would be met with functional adaptations in affected areas and those downstream in order to minimize the cognitive impact of the brain changes. Such a compensatory mechanistic interpretation is consistent with much empirical data (e.g., [53]). This observation is comparable to that in neural reserve and compensation, which encompass similar mechanisms, including 'compensatory' networks that are associated with poorer performance [54].

Proponents of STAC suggest that it differs from CR in that it applies not only to aging, but also to the brain's response to other pathologies or afflictions throughout the lifespan, as well as to normal task-related challenges, such as learning a new skill. [14] However, this overlooks the fact that CR is built up over a lifetime of experiences [55] and has been associated with reduced susceptibility to many forms of brain damage across the lifespan [56]. CR

\section{Box 3. Cognitive manifestations of CR}

Despite the overwhelming emphasis on the neural underpinnings of reserve, Lövdén et al.'s [51] conceptual work on cognitive flexibility as a counterpart to neural plasticity should remind us that there is a purely behavioral dimension to $C R$ that is based solely on cognitive networks - that is, acquired knowledge or 'representations' that do not reflect structural differences beyond synaptic connectivity.

These behavioral manifestations of $\mathrm{CR}$ may be reflected, for instance, in an increased ability to select and employ the best strategy for performing a task. Barulli et al. [57] report that higher CR (operationalized using verbal $\mathrm{IQ}$ and years of education) in healthy adults is associated with better strategy selection abilities in a computational estimation task: older adults with higher verbal IQs employed the best strategy more often than those with lower verbal 10 , an effect not observed in a young group. This finding suggests that it is not overall cognitive ability which determines strategy selection, but $\mathrm{CR}$ based on a lifetime of acquired knowledge.

Other more specific cognitive domains - particularly memory, where mnemonic strategies would be very effective $[88,89]-$ may also be useful for evaluating the cognitive implementation of reserve. Woods et al. [90] report that in a sample of HIV-infected participants mnemonic strategy use was associated with higher verbal $\mathrm{IO}$ and moderated the effects of the disease on visual working memory. There is also a growing body of evidence to suggest that higher CR is associated with better use of compensatory strategies. For example, Czernochowski et al. [91] found that higher-socioeconomic status (SES) older adults had access to compensatory mnemonic strategies that lower-SES elders did not. Boyle et al. [92] reported that poor decision-making was associated with a fourfold increased risk of mortality in a sample of 675 older adults, even after controlling for other cognitive domains. Whether improved strategy selection or decision-making abilities are an instantiation of CR or a basis for it is an open question.

Although very preliminary, these results suggest one largely unexplored path to studying the cognitive mechanisms of $\mathrm{CR}$ and related concepts. Identifying the particular cognitive strategies or compensatory mechanisms may help to identify the underlying neural mechanisms, as well as point towards effective interventions. 
has also been used to predict discrepancies in performance in the case of normal aging (e.g., [57]), as well as differences in performance and functional MRI activation resulting from differences in task difficulty (e.g., [58]). Moreover, in a longitudinally-followed sample of young people, low childhood IQ was found to be predictive of increased risk for psychiatric disorders, such as depression, schizophrenia, and anxiety in young adulthood [59]. This suggests that CR may be important throughout the lifespan for resisting not only cognitive decline, but psychiatric afflictions, as well. Thus, CR is a model with wide applicability across the lifespan and across a range of brain challenges, but despite its generality it can also account for qualitative shifts that occur in compensatory behavior (e.g., shifts in functional activation upon diagnosis, dramatic shifts in the rate of cognitive decline, etc.) in a way that a more generalized theory such as scaffolding cannot.

\section{Concluding remarks}

In this review, we attempted to clarify some of the conceptual relationships between CR and closely related models. As imaging methods become more advanced and macrostructural changes reveal their microstructural correlates, BR and CR may grow more interconnected. Much more fine-grained brain measures are necessary than the standard proxies for BR, such as brain size, and CR can point towards which of these more subtle measures are relevant. $\mathrm{BM}$ theories, meanwhile, are complementary to CR, but it is not clear to what extent they can account for age-related variability in cognitive performance, given their inability to explain compensatory mechanisms. Even with normal aging, some degree of structural change seems to be inevitable; therefore, these changes present a challenge with which the healthy ager needs to cope. Finally, scaffolding and related theories are also compatible with CR and, we

\section{Box 4. Outstanding questions}

- What are the neural mechanisms by which CR operates? To what extent are they captured by the neural reserve and neural compensation hypotheses?

- Could there be a general 'CR network' - that is, a network developed over a lifetime of cognitive stimulation that is active and playing a compensatory role across very disparate tasks?

- How do CR and BR interact? When and how is cognitive experience converted to structural change?

- Can life experiences foster BM? Would this apply only to normal aging or to disease pathologies, such as plaques and tangles?

- Can experiences be provided that impart CR?

- To what extent is CR based on acquired knowledge (analogous to crystallized intelligence) versus cognitive processes (analogous to fluid intelligence)?

- What role do genetic factors play in CR and BR? Do genetic factors merely set the initial limits for cognitive flexibility or could they also set limits for neuroplastic structural changes?

- Might CR be easier to build at younger ages, consistent with the observation in animal studies of 'critical periods' [93,94], during which certain neuroplastic changes are more likely?

- How much normal age-related cognitive variance is accounted for by BM theories and CR theories, respectively? Is this question possible to answer without a clearer conceptual distinction between what constitutes normal aging and what constitutes pathology? have suggested, propose many similar overarching principles and mechanisms. The challenge for all theories now is to specify the underlying neural and cognitive mechanisms that mediate the relationship between brain challenge and cognitive performance (Box 4).

\section{Acknowledgements}

Supported by NIH/NIA RO1 AG26158.

\section{References}

1 Stern, Y. (2002) What is cognitive reserve? Theory and research application of the reserve concept. J. Int. Neuropsychol. Soc. 8, 448-460

2 Stern, Y. (2009) Cognitive reserve. Neuropsychologia 47, 2015-2028

3 Stern, Y. et al. (1994) Influence of education and occupation on the incidence of Alzheimer's disease. J. Am. Med. Assoc. 271, 1004-1010

4 Scarmeas, N. et al. (2001) Influence of leisure activity on the incidence of Alzheimer's disease. Neurology 57, 2236-2242

5 Wilson, R.S. (2009) Elderly women with larger social networks are less likely to develop dementia. Evid. Based Ment. Health 12, 22

6 Valenzuela, M.J. and Sachdev, P. (2005) Brain reserve and dementia: a systematic review. Psychol. Med. 25, 1-14

7 Suchy, Y. et al. (2011) Instrumental activities of daily living among community-dwelling older adults: discrepancies between self-report and performance are mediated by cognitive reserve. J. Clin. Exp. Neuropsychol. 33, 92-100

8 Fay, T.B. et al. (2010) Cognitive reserve as a moderator of postconcussive symptoms in children with complicated and uncomplicated mild traumatic brain injury. J. Int. Neuropsychol. Soc. 16, 94-105

9 Poletti, M. et al. (2011) Mild cognitive impairment and cognitive reserve in Parkinson's disease. Parkinsonism Relat. Disord. 17, 579-586

10 Sumowski, J.F. et al. (2009) Cognitive reserve protects against cognitive dysfunction in multiple sclerosis. J. Clin. Exp. Neuropsychol. 31, 913-926

11 Foley, J.M. et al. (2012) Cognitive reserve as a protective factor in older HIV-positive patients at risk for cognitive decline. Appl. Neuropsychol. $19,16-25$

12 Satz, P. et al. (2011) Brain and cognitive reserve: mediator(s) and construct validity, a critique. J. Clin. Exp. Neuropsychol. 33, 121-130

13 Nyberg, L. et al. (2012) Memory aging and brain maintenance. Trends Cogn. Sci. 16, 292-305

14 Park, D.C. and Reuter-Lorenz, P. (2009) The adaptive brain: aging and neurocognitive scaffolding. Annu. Rev. Psychol. 60, 173-196

15 Sachdev, P.S. and Valenzuela, M. (2009) Brain and cognitive reserve. Am. J. Geriatr. Psychiatry 17, 175-178

16 Mortimer, J.A. et al. (2003) Head circumference, education and risk of dementia: findings from the Nun Study. J. Clin. Exp. Neuropsychol. 25, $671-679$

17 Graves, A.B. et al. (2001) Head circumference and incident Alzheimer's disease: modification by apolipoprotein E. Neurology 57, 1453-1460

18 Schofield, P.W. et al. (1997) An association between head circumference and Alzheimer's disease in a populationbased study of aging. Neurology 49, 30-37

19 Wang, Y. et al. (2012) Selective changes in white matter integrity in MCI and older adults with cognitive complaints. Biochim. Biophys. Acta 1822, 423-430

20 Chao, L.L. et al. (2013) Associations between white matter hyperintensities and $\beta$ amyloid on integrity of projection, association, and limbic fiber tracts measured with diffusion tensor MRI. PLOS ONE 8, e65175

21 Holtmaat, A. and Svoboda, K. (2009) Experience-dependent structural synaptic plasticity in the mammalian brain. Nat. Rev. Neurosci. 10, 647-658

22 Lövdén, M. et al. (2013) Structural brain plasticity in adult learning and development. Neurosci. Biobehav. Rev. http://dx.doi.org/10.1016/ j.neubiorev.2013.02.014

23 Lerch, J.P. et al. (2011) Maze training in mice induces MRI-detectable brain shape changes specific to the type of learning. Neuroimage 54, 2086-2095

24 Jones, R.N. et al. (2011) Conceptual and measurement challenges in research on cognitive reserve. J. Int. Neuropsychol. Soc. 17, 593-601 
25 Reed, B.R. et al. (2010) Measuring cognitive reserve based on the decomposition of episodic memory variance. Brain 133, 2196-2209

26 Yaffe, K. et al. (2011) Association of plasma $\beta$-amyloid level and cognitive reserve with subsequent cognitive decline. JAMA 305, 261-266

27 Ewers, M. et al. (2013) Cognitive reserve associated with FDG-PET in preclinical Alzheimer disease. Neurology 80, 1194-1201

28 Ahles, T.A. et al. (2010) Longitudinal assessment of cognitive changes associated with adjuvant treatment for breast cancer: impact of age and cognitive reserve. J. Clin. Oncol. 28, 4434-4440

29 Solé-Padullés, C. et al. (2009) Brain structure and function related to cognitive reserve variables in normal aging, mild cognitive impairment and Alzheimer's disease. Neurobiol. Aging 30, 1114-1124

30 Bosch, B. et al. (2010) Cognitive reserve modulates task-induced activations and deactivations in healthy elders, amnestic mild cognitive impairment and mild Alzheimer's disease. Cortex 46, 451461

31 Stern, Y. (2006) Cognitive reserve and Alzheimer disease. Alzheimer Dis. Assoc. Disord. 20, S69-S74

32 Neubauer, A.C. and Fink, A. (2009) Intelligence and neural efficiency. Neurosci. Biobehav. Rev. 33, 1004-1023

33 Fabiani, M. (2012) It was the best of times, it was the worst of times: a psychophysiologist's view of cognitive aging. Psychophysiology 49, 283 304

34 van Praag, H. et al. (1999) Running enhances neurogenesis, learning, and long-term potentiation in mice. Proc. Natl. Acad. Sci. U.S.A. 96, 13427-13431

35 Aguiar, A.S., Jr et al. (2011) Short bouts of mild-intensity physical exercise improve spatial learning and memory in aging rats: involvement of hippocampal plasticity via AKT, CREB and BDNF signaling. Mech. Ageing Dev. 132, 560-567

36 Cotman, C.W. and Berchtold, N.C. (2002) Exercise: a behavioral intervention to enhance brain health and plasticity. Trends Neurosci. 25, 295-301

37 Nithianantharajah, J. and Hannan, A.J. (2009) The neurobiology of brain and cognitive reserve: mental and physical activity as modulators of brain disorders. Prog. Neurobiol. 89, 369-382

38 Coffey, C.E. et al. (1999) Relation of education to brain size in normal aging: implications for the reserve hypothesis. Neurology 53, 189-196

39 Gaser, C. and Schlaug, G. (2003) Brain structures differ between musicians and non-musicians. J. Neurosci. 23, 9240-9245

40 Carreiras, M. et al. (2009) An anatomical signature for literacy. Nature 461, 983-986

41 Erickson, K.I. et al. (2011) Exercise training increases size of hippocampus and improves memory. Proc. Natl. Acad. Sci. U.S.A. 108, 3017-3022

42 Draganski, B. and May, A. (2008) Training-induced structural changes in the adult human brain. Behav. Brain Res. 192, 137-142

43 Woollett, K. and Maguire, E.A. (2011) Acquiring 'the Knowledge' of London's layout drives structural brain changes. Curr. Biol. 21, 21092114

44 Valenzuela, M.J. et al. (2008) Lifespan mental activity predicts diminished rate of hippocampal atrophy. PLOS ONE 3, e2598

45 Jagust, W.J. and Mormino, E.C. (2011) Lifespan brain activity, betaamyloid, and Alzheimer's disease. Trends Cogn. Sci. 15, 520-526

46 Boyle, P.A. et al. (2013) Much of late life cognitive decline is not due to common neurodegenerative pathologies. Ann. Neurol. http:// dx.doi.org/10.1002/ana.23964

47 Brayne, C. et al. (2010) Education, the brain and dementia: neuroprotection or compensation? EClipSE Collaborative Members. Brain 133, 2210-2216

48 Wenger, E. et al. (2012) Cortical thickness changes following spatial navigation training in adulthood and aging. Neuroimage 59, 33893397

49 Mandolesi, L. et al. (2008) Environmental enrichment provides a cognitive reserve to be spent in the case of brain lesion. $J$. Alzheimers Dis. 15, 11-28

50 Reuter-Lorenz, P.A. and Cappell, K.A. (2008) Neurocognitive aging and the compensation hypothesis. Curr. Dir. Psychol. Sci. 17, 177-182

51 Lövdén, M. et al. (2010) A theoretical framework for the study of adult cognitive plasticity. Psychol. Bull. 136, 659-676
52 Shing, Y.L. et al. (2008) Associative and strategic components of episodic memory: a life-span dissociation. J. Exp. Psychol. Gen. 137, $495-513$

$53 \mathrm{Ilg}$, R. et al. (2008) Gray matter increase induced by practice correlates with task-specific activation: a combined functional and morphometric magnetic resonance imaging study. J. Neurosci. 28, 4210-4215

54 Steffener, J. et al. (2008) The impact of structure on age-related changes in working memory functional activity. Brain Imaging Behav. 3, 142-153

55 Valenzuela, M.J. and Sachdev, P. (2007) Assessment of complex mental activity across the lifespan: development of the Lifetime of Experiences Questionnaire (LEQ). Psychol. Med. 37, 1015-1026

56 Katzman, R. (1993) Education and the prevalence of dementia and Alzheimer's disease. Neurology 43, 13-20

57 Barulli, D.J. et al. (2013) The influence of cognitive reserve on strategy selection in normal aging. J. Int. Neuropsychol. Soc. 19, 1-4

58 Stern, Y. et al. (2012) Task difficulty modulates young-old differences in network expression. Brain Res. 1435, 130-145

59 Koenen, K.C. et al. (2009) Childhood IQ and adult mental disorders: a test of the cognitive reserve hypothesis. Am. J. Psychiatry 166, 50-57

60 Amieva, H. et al. (2005) The 9 year cognitive decline before dementia of the Alzheimer type: a prospective population-based study. Brain 128 , 1093-1101

61 Hall, C.B. et al. (2007) Education delays accelerated decline on a memory test in persons who develop dementia. Neurology 69, 16571664

62 Stern, Y. et al. (1992) Inverse relationship between education and parietotemporal perfusion deficit in Alzheimer's disease. Ann. Neurol. 32, 371-375

63 Stern, Y. et al. (1999) Rate of memory decline in AD is related to education and occupation: Cognitive reserve? Neurology 53, 1942-1957

64 Steffener, J. et al. (2011) Supporting performance in the face of agerelated neural changes: testing mechanistic roles of cognitive reserve. Brain Imaging Behav. 22, 655-669

65 Stern, Y. (2012) Cognitive reserve in ageing and Alzheimer's disease. Lancet Neurol. 11, 1006-1012

66 Erickson, K. and Kramer, A.F. (2009) Aerobic exercise effects on cognitive and neural plasticity in older adults. Br. J. Sports Med. 43, 22-24

67 Smith, P.J. et al. (2010) Aerobic exercise and neurocognitive performance: a meta-analytic review of randomized controlled trials. Psychosom. Med. 72, 239-252

68 Basak, C. et al. (2008) Can training in a real-time strategy video game attenuate cognitive decline in older adults? Psychol. Aging 23, 765-777

69 Stern, Y. et al. (2011) Space Fortress game training and executive control in older adults: a pilot intervention. Neuropsychol. Dev. Cogn. $18,653-677$

70 Owen, A.M. et al. (2010) Putting brain training to the test. Nature 465, $775-778$

71 Jaeggi, S.M. et al. (2008) Improving fluid intelligence with training on working memory. Proc. Natl. Acad. Sci. U.S.A. 105, 6829-6833

72 Edwards, J.D. et al. (2009) The longitudinal impact of cognitive speed of processing training on driving mobility. Gerontologist 49, 485-494

73 Lovden, M. et al. (2010) Experience-dependent plasticity of whitematter microstructure extends into old age. Neuropsychologia 48, 3878-3883

74 Engvig, A. et al. (2012) Memory training impacts short-term changes in aging white matter: a longitudinal diffusion tensor imaging study. Hum. Brain Mapp. 33, 2390-2406

75 Engvig, A. et al. (2010) Effects of memory training on cortical thickness in the elderly. Neuroimage 52, 1667-1676

76 Scholz, J. et al. (2009) Training induces changes in white-matter architecture. Nat. Neurosci. 12, 1370-1371

77 Colom, R. et al. (2012) Structural changes after videogame practice related to a brain network associated with intelligence. Intelligence 40 , 479-489

78 Takeuchi, H. et al. (2010) Training of working memory impacts structural connectivity. J. Neurosci. 30, 3297-3303

79 Schmidt-Wilcke, T. et al. (2010) Distinct patterns of functional and structural neuroplasticity associated with learning Morse code. Neuroimage 51, 1234-1241 
80 Kwok, V. et al. (2011) Learning new color names produces rapid increase in gray matter in the intact adult human cortex. Proc. Natl. Acad. Sci. U.S.A. 108, 6686-6688

81 Sagi, Y. et al. (2012) Learning in the fast lane: new insights into neuroplasticity. Neuron 73, 1195-1203

82 Steffener, J. and Stern, Y. (2012) Exploring the neural basis of cognitive reserve in aging. Biochim. Biophys. Acta 1822, 467-473

83 Schneider-Garces, N.J. et al. (2009) Span, CRUNCH, and Beyond: Working Memory Capacity and the Aging Brain. J. Cogn. Neurosci. 22, 655-669

84 Davis, S.W. et al. (2008) Que PASA? The posterior-anterior shift in aging. Cereb. Cortex 18, 1201-1209

85 Berlingeri, M. et al. (2013) Reassessing the HAROLD model: is the hemispheric asymmetry reduction in older adults a special case of compensatory-related utilisation of neural circuits? Exp. Brain Res. 224, 393-410

86 Rossi, S. et al. (2004) Age-related functional changes of prefrontal cortex in long-term memory: a repetitive transcranial magnetic stimulation study. J. Neurosci. 24, 7939-7944

87 Stern, Y. et al. (2008) A common neural network for cognitive reserve in verbal and object working memory in young but not old. Cereb. Cortex $18,959-967$
88 Garrett, D.D. et al. (2010) Everyday memory compensation: the impact of cognitive reserve, subjective memory, and stress. Psychol. Aging 25, $74-83$

89 Uttner, I. et al. (2010) Reduced benefit from mnemonic strategies in early-stage Alzheimer's disease: a brief testing-the-limits paradigm for clinical practice. J. Neurol. 257, 1718-1726

90 Woods, S.P. et al. (2010) Spontaneous strategy use protects against visual working memory deficits in older adults infected with HIV. Arch. Clin. Neuropsychol. 25, 724-733

91 Czernochowski, D. et al. (2008) Use it or lose it? SES mitigates agerelated decline in a recency/recognition task. Neurobiol. Aging 29, 945958

92 Boyle, P.A. et al. (2013) Poor decision making is associated with an increased risk of mortality among community-dwelling older persons without dementia. Neuroepidemiology 40, 247-252

93 Wagner, A. et al. (2000) Brain plasticity: to what extent do aged animals retain the capacity to coordinate gene activity in response to acute challenges. Exp. Gerontol. 35, 1211-1227

94 Bloss, E.B. et al. (2011) Evidence for reduced experience-dependent dendritic spine plasticity in the aging prefrontal cortex. J. Neurosci. 31, 7831-7839 